\title{
Insulin resistance influences the association of adiponectin levels with diabetes incidence in two population-based cohorts: the Cooperative Health Research in the Region of Augsburg (KORA) S4/F4 study and the Framingham Offspring Study
}

\author{
M.-F. Hivert • L. M. Sullivan • P. Shrader • C. S. Fox • D. M. Nathan • \\ R. B. D'Agostino Sr • P. W. F. Wilson • B. Kowall • C. Herder • C. Meisinger • \\ B. Thorand $\cdot$ W. Rathmann $\cdot$ J. B. Meigs
}

Received: 21 June 2010 / Accepted: 2 January 2011 /Published online: 19 February 2011

(C) Springer-Verlag 2011

\begin{abstract}
Aims/hypothesis Lower adiponectin levels are associated with higher risk of incident type 2 diabetes. Most analyses have been adjusted for confounding factors, but few have taken into account insulin resistance per se. We tested the hypothesis that the association of adiponectin levels with incident type 2 diabetes differs between insulin-resistant and insulin-sensitive individuals.

Methods We studied two prospective cohorts: the Framingham Offspring Study $(n=2,023)$ and the Cooperative
\end{abstract}

Electronic supplementary material The online version of this article (doi:10.1007/s00125-011-2067-y) contains supplementary material, which is available to authorised users.

M.-F. Hivert · D. M. Nathan · J. B. Meigs

Department of Medicine, Harvard Medical School,

Boston, MA, USA

M.-F. Hivert $\cdot$ P. Shrader $\cdot$ J. B. Meigs $(\bowtie)$

General Medicine Division, Massachusetts General Hospital,

50 Staniford Street, 9th Floor,

Boston, MA 02114, USA

e-mail: jmeigs@partners.org

L. M. Sullivan

Department of Biostatistics,

Boston University School of Public Health,

Boston, MA, USA

C. S. Fox

The National Heart, Lung, and Blood Institute's Framingham Heart Study,

Framingham, MA, USA
Health Research in the Region of Augsburg (KORA) S4/F4 study $(n=887)$ cohorts. Insulin resistance was estimated by HOMA-insulin resistance (HOMA-IR). We used logistic regression analysis to test the association between adiponectin and incident type 2 diabetes overall and in insulin-resistant vs insulin-sensitive individuals (defined by $\geq$ vs $<75$ th percentile of HOMA-IR).

Results At baseline, Framingham's participants were $60 \pm$ 9 years old and $56 \%$ were women; KORA's participants were $63 \pm 5$ years old and $49 \%$ were women. Type 2

\section{S. Fox}

Department of Endocrinology and Metabolism,

Brigham and Women's Hospital,

Boston, MA, USA

D. M. Nathan

Diabetes Center, Department of Medicine,

Massachusetts General Hospital,

Boston, MA, USA

R. B. D'Agostino Sr

Department of Mathematics and Statistics/Consulting Unit,

Boston University,

Boston, MA, USA

P. W. F. Wilson

Emory Clinical Cardiovascular Research Institute,

Emory University School of Medicine,

Atlanta, GA, USA 
diabetes incidence was $5.4 \%$ over 6.5 years $(n=109)$ in Framingham and $10.5 \%$ over 8 years $(n=93)$ in KORA. Lower adiponectin levels were associated with type 2 diabetes incidence in both cohorts. In insulin-resistant individuals, lower adiponectin levels were associated with higher risk of type 2 diabetes incidence (OR 1.60 [95\% CI 1.10-2.31] per SD decrease in Framingham, $p=0.01$; and OR 2.34 [95\% CI 1.16-4.73] in KORA, $p=0.02$ ); while this was not observed in insulin-sensitive individuals (OR 1.10 [95\% CI 0.73-1.67] in Framingham, $p=0.64$; and OR 1.34 [95\%CI: 0.88-2.03] in KORA, $p=0.18$ ).

Conclusions/interpretation We conclude that lower adiponectin levels are associated with higher risk of type 2 diabetes in insulin-resistant but not in insulin-sensitive individuals. This suggests that some level of insulin resistance is needed to see deleterious effects of low adiponectin.

Keywords Adiponectin · Insulin resistance $\cdot$ Diabetes incidence $\cdot$ Prospective cohorts

$\begin{array}{ll}\text { Abbreviations } \\ \text { HOMA-IR } & \text { HOMA-insulin resistance } \\ \text { KORA } & \text { Cooperative Health Research in the Region of } \\ & \text { Augsburg }\end{array}$

\section{Introduction}

Many adipokines produced by the adipose tissue are hypothesised to relate to type 2 diabetes pathophysiology, linking excess weight and development of the disease. One of the adipokines of interest is adiponectin. In contrast to other adipokines, adiponectin levels are inversely correlated with adiposity and insulin resistance in most animal and human studies. Moreover, prospective human studies have demonstrated that low adiponectin levels are associated with an increased risk of developing diabetes [1]. Genetic variants in the gene that encodes adiponectin $(A D I P O Q)$ are associated with hyperglycaemia [2]. Based on those reports, some have suggested that low adiponectin levels are a

\footnotetext{
B. Kowall $\cdot$ W. Rathmann

Institute of Biometrics and Epidemiology,

German Diabetes Center, Leibniz Center for Diabetes Research

at Heinrich Heine University,

Düsseldorf, Germany

C. Herder

Institute for Clinical Diabetology, German Diabetes Center,

Leibniz Center for Diabetes Research at Heinrich Heine University, Düsseldorf, Germany

C. Meisinger $\cdot$ B. Thorand

Institute of Epidemiology II, Helmholtz Zentrum München,

Neuherberg, Germany
}

primary defect leading to insulin resistance and the development of diabetes, but this needs further investigation in humans.

We tested the hypothesis that low adiponectin is associated with increased risk of type 2 diabetes incidence in two well-characterised prospective cohorts, those of the Framingham Offspring Study and the Cooperative Health Research in the Region of Augsburg (KORA) S4/F4 study. We explored the influence of insulin resistance on the association between adiponectin and diabetes incidence by adjusting for levels of insulin resistance and by stratifying the population into insulin-resistant vs insulin-sensitive individuals.

\section{Methods}

Framingham Offspring Study The Framingham Offspring Study is a community-based prospective observational study that has followed the children of the original Framingham Heart Study cohort and their spouses [3]. At baseline in 1971, 5,124 participants of European ancestry were recruited. For this analysis, we used the follow-up from examination cycle 7 (1998-2001) to cycle 8 (20032007): a total of 2,023 participants free of diabetes at baseline and with adipokine measurements completed were included in the present report. The study protocol was approved by the Institutional Review Boards of the Boston University Medical Center and of the Massachusetts General Hospital; all participants provided written informed consent.

At every examination cycle, participants had a detailed medical history and physical examination, standardised anthropometric measurements, and fasting blood samples. BMI was calculated as the weight in $\mathrm{kg}$ divided by the square of height in metres $\left(\mathrm{kg} / \mathrm{m}^{2}\right)$. Diabetes was defined as use of diabetes medication or a fasting plasma glucose $\geq 7.0 \mathrm{mmol} / 1$ at the index examination. Insulin resistance was defined by the HOMA-insulin resistance (HOMA-IR) formula [4]. Fasting plasma glucose was measured immediately with a hexokinase reagent kit (A-gent glucose test; Abbott, South Pasadena, CA, USA), and other plasma samples were frozen at $-80^{\circ} \mathrm{C}$ until assay. Fasting plasma insulin was measured with a human specific-insulin assay, having essentially no cross-reactivity to proinsulin or insulin split products (Linco, St. Louis, MO, USA). Total adiponectin levels were measured by ELISA (R\&D Systems, Minneapolis, MN, USA); other adipokine levels (resistin and TNF- $\alpha$ ), available at examination 7 , were also measured by ELISA (R\&D Systems). Intra-assay coefficients of variation were $<3 \%$ for glucose, $6.1 \%$ for insulin, $5.8 \%$ for adiponectin, $9.0 \%$ for resistin and $6.6 \%$ for TNF- $\alpha$. 
KORA S4/F4 cohort study The study sample was 2,656 individuals aged 55-74 years living in the city of Augsburg (Germany) and in 16 towns and villages from the surrounding districts in 1999 (600,000 inhabitants) [5]. Informed consent was obtained from the participants. Overall, 1,653 (62\%) individuals participated. After excluding those with known diabetes and further dropouts, 1,353 participants had an OGTT at baseline. This cohort was re-investigated in 2006 to 2008 [6]. The present study includes only participants without known or newly diagnosed diabetes at baseline, who successfully completed a baseline OGTT and who lived within the study region at the time of the follow-up examination $(n=1,202)$. Among those individuals, 98 (8\%) had deceased by 2006, and $887(74 \%)$ participated in the follow-up (OGTT).

Cases of incident diabetes (self-reports) and the dates of diagnosis were validated by contacting the general practitioners who treated the participants. Among all other participants, OGTT was performed during the morning hours after overnight fasting. Fasting venous blood glucose was sampled and $75 \mathrm{~g}$ of anhydrous glucose was given (Dextro OGT; Boehringer, Mannheim, Germany). Incident diabetes was defined based on: (1) validated physician diagnosis; or (2) newly diagnosed diabetes ( $\geq 7.0 \mathrm{mmol} / \mathrm{l}$ fasting or $\geq 11.1 \mathrm{mmol} / 1$ for $2 \mathrm{~h}$ glucose).

Body weight was measured in light clothing by trained investigators to the nearest $0.1 \mathrm{~kg}$, and height to the nearest $0.5 \mathrm{~cm}$. Waist circumference was measured at the minimum abdominal girth to the nearest $0.1 \mathrm{~cm}$. Blood pressure was measured by quality-certified study investigators three times at the right arm in a sitting position after a 15 min rest using a validated automatic device. The mean of the second and third measurements was used for the analysis. Family diabetes history was assessed in a structured interview.

Blood was collected with minimal stasis, refrigerated at $4-8^{\circ} \mathrm{C}$ and shipped in refrigerant packaging within $2-4 \mathrm{~h}$ to the laboratory of Augsburg Central Hospital. Blood glucose was measured using a hexokinase method (Gluco-quant; Roche Diagnostics, Mannheim, Germany). At baseline, the inter-assay coefficients of variation for glucose were $2.4 \%$ at $5.4 \mathrm{mmol} / 1$ and $2.1 \%$ at $13 \mathrm{mmol} / 1$. Serum adiponectin concentration was measured using the radioimmunoassay from Linco. Mean intra- and inter-assay variations in control sera were $5.5 \%$ and $9.2 \%$, respectively [7]. Fasting insulin was determined using a microparticle enzyme immunoassay (Abbott). The insulin resistance score (HOMA-IR) was calculated [4].

Statistical analysis Baseline characteristics are presented as means $( \pm \mathrm{SD})$ for continuous variables, or percentages $(\%)$ for categorical variables. Spearman correlation was used to test the association between HOMA-IR and adiponectin levels. Multivariable logistic regression analysis was used to analyse the association between diabetes incidence and variables, including the primary exposure of interest: adiponectin levels. Because of its significant association with adiponectin levels and the putative insulin-sensitising function of adiponectin, we stratified our population according to insulin resistance state measured by HOMA-IR. By convention, we defined insulin resistant as HOMA-IR $\geq 75$ th percentile and insulin sensitive as HOMA-IR $<75$ th percentile (the 75th percentile within each cohort was used to define insulin-resistant vs insulin-sensitive participants).

Using Framingham Offspring Study data, we conducted secondary analyses: we adjusted for baseline levels of other adipokines (resistin or TNF- $\alpha$ ), and for waist circumference or visceral adipose tissue (VAT; measured by computerised tomography, $n=921$ ) instead of BMI to assess the impact of central adiposity. Finally, we used metabolic syndrome as a proxy for insulin resistance instead of HOMA-IR in the main and stratified analyses. Metabolic syndrome was defined according to the National Cholesterol Education Program Adult Treatment Program III (NCEP-ATP3) definition [8]. We considered $p$ values less than 0.05 to indicate statistical significance. We performed all analyses using SAS software (version 8.1, SAS Institute, Cary, NC, USA in Framingham Offspring Study; and version 9.2, SAS Institute, Cary, NC, USA in KORA).

\section{Results}

The baseline characteristics of participants are presented in Table 1. Despite being clinically similar to each other, participants from KORA were statistically older $(p<0.0001)$, had a slightly higher BMI $(p=0.04)$, and were more likely to have a family history of type 2 diabetes $(p=0.001)$.

Over the 6.5 year follow-up in the Framingham Offspring Study, 5.4\% of participants $(n=109)$ developed type 2 diabetes. Lower adiponectin levels were associated with an increased risk of diabetes, even after adjustment for age, sex and BMI (OR 1.87 [95\% CI 1.36-2.57] per SD decrease in adiponectin levels; $p=0.0001$, see Table 2). Consistent with our previous report [9], baseline adiponectin levels were inversely associated with insulin resistance measured by HOMA-IR (Spearman $r=-0.40 ; p<0.0001$ ). Further adjustment for HOMA-IR in multivariable models predicting diabetes incidence reduced the effect of adiponectin, but the association remained significant (OR 1.44 [95\% CI 1.04-1.97]; $p=0.03$, see Table 1). Over 8 years of follow-up in KORA, the incidence of diabetes was $10.5 \%$ (93 cases). After adjustment for age, sex, BMI and HOMAIR, lower adiponectin levels were associated with increased risk of diabetes in KORA participants, (OR 1.71 [95\% CI 1.19-2.45] per SD decrease in adiponectin levels; $p=0.004$, see Table 2). 
Table 1 Baseline characteristics of participants free of diabetes in the Framingham Offspring Study (1998-2001) and in the KORA S4/F4 study (1999-2001)

\begin{tabular}{|c|c|c|}
\hline Characteristic & $\begin{array}{l}\text { Framingham Offspring } \\
\text { Study }(n=2,023)\end{array}$ & $\begin{array}{l}\text { KORA } \\
(n=887)\end{array}$ \\
\hline Age (years) & $59.6(9.1)$ & $63.2(5.4)$ \\
\hline Men, $n(\%)$ & $893(44.1)$ & $449(50.6)$ \\
\hline $\begin{array}{l}\text { Family history of diabetes, } \\
n(\%)\end{array}$ & $342(16.9)$ & $203(22.9)$ \\
\hline BMI $\left(\mathrm{kg} / \mathrm{m}^{2}\right)$ & $27.7(5.0)$ & $28.1(4.0)$ \\
\hline Waist circumference $(\mathrm{cm})$ & $98.3(13.5)$ & $94.5(11.1)$ \\
\hline HDL-cholesterol (mmol/l) & $1.43(0.44)$ & $1.53(0.42)$ \\
\hline Triacylglycerol (mmol/l) & $1.46(0.88)$ & $1.44(0.80)$ \\
\hline Fasting glucose $(\mathrm{mmol} / \mathrm{l})$ & $5.39(0.53)$ & $5.47(0.52)$ \\
\hline $\mathrm{SBP}(\mathrm{mmHg})$ & $125.1(18.2)$ & $133.1(18.8)$ \\
\hline DBP (mmHg) & $74.3(9.6)$ & $79.8(10.2)$ \\
\hline $\begin{array}{l}\text { Fasting plasma insulin } \\
(\mathrm{pmol} / 1)^{\mathrm{a}}\end{array}$ & $82.9(47.9)$ & $91.7(121.6)$ \\
\hline HOMA-IR $^{\mathrm{a}}$ & $3.7(2.5)$ & $3.3(4.2)$ \\
\hline Adiponectin $(\mathrm{mg} / \mathrm{ml})^{\mathrm{a}}$ & $8.8(1.8)$ & $10.0(5.5)$ \\
\hline
\end{tabular}

Values are mean (SD) unless stated otherwise

${ }^{a}$ In KORA, median (IQR) values for fasting insulin=67.2 pmol/1 (47.9-97.9 pmol/1); for HOMA-IR=2.4 (1.6-3.5); for adiponectin= $9.0 \mathrm{mg} / \mathrm{ml}(6.2-12.3 \mathrm{mg} / \mathrm{ml})$

DBP, diastolic blood pressure; SBP, systolic blood pressure

Next, we tested the association of adiponectin levels and diabetes incidence after stratification of participants as insulin resistant (HOMA-IR $\geq 75$ th percentile) and insulin sensitive (HOMA-IR $<75$ th percentile; see Table 2). In insulin-resistant participants, low adiponectin levels were associated with higher risk of diabetes (OR 1.60 [95\% CI 1.10-2.31] per SD decrease in Framingham, $p=0.01$; and OR 2.34 [95\% CI 1.16-4.73] in KORA. $p=0.02$ ), while this was not observed in insulin-sensitive participants (OR
1.10 [95\% CI 0.73-1.67] in Framingham, $p=0.64$; OR 1.34 [95\% CI $0.88-2.03]$ in KORA; $p=0.18$ ). The interaction between adiponectin levels and insulin-resistant state was statistically significant in KORA $(p=0.045)$ but not in the Framingham Offspring Study $(p=0.41)$. We also tested the interaction between adiponectin and HOMA-IR treated as continuous variables in Framingham (see electronic supplementary material [ESM] Table 1); although both lower adiponectin levels and higher HOMA-IR were significantly associated with higher risk of diabetes incidence, the interaction was not significant $(p=0.25$, adjusted for age, sex, BMI). Diabetes incidence in each tertile of baseline adiponectin levels in insulin-resistant vs insulin-sensitive participants is illustrated for Framingham in Fig. 1 and for KORA in Fig. 2.

Subsidiary analyses in Framingham Offspring Study Further adjustment of our main analyses with resistin or TNF- $\alpha$ levels measured at baseline did not modify the association between adiponectin levels and type 2 diabetes incidence (OR 1.43 [95\% CI 1.04-1.97]; $p=0.03$ ), indicating that the association is independent of other adipokines previously shown to be associated with insulin resistance in the Framingham Offspring Study [9]. Adjusting for waist circumference (OR 1.42 [95\% CI 1.03-1.95]; $p=0.03$ ) or VAT (OR 1.38 [95\% CI $0.88-2.15] ; p=0.15$ ) instead of BMI did not substantially influence the OR (but the $p$ value after adjustment for VAT was no longer significant, driven by smaller sample size $n=921$ ). Finally, as a sensitivity analysis, we used metabolic syndrome to classify individuals at higher metabolic risk associated with insulin resistance: the association between adiponectin level and type 2 diabetes incidence remained significant even when adjusting for metabolic syndrome rather than HOMA-IR. In the group with metabolic syndrome there was a significant association between lower adiponectin and higher risk of

Table 2 Multivariable logistic regression models predicting diabetes incidence in participants of the Framingham Offspring Study and KORA S4/F4 study

\begin{tabular}{|c|c|c|c|c|c|c|}
\hline \multirow[t]{2}{*}{ Variable } & \multicolumn{3}{|c|}{ Framingham Offspring Study } & \multicolumn{3}{|l|}{ KORA } \\
\hline & $\begin{array}{l}\text { OR for type } 2 \\
\text { diabetes }\end{array}$ & $95 \% \mathrm{CI}$ & $p$ value & $\begin{array}{l}\text { OR for type } 2 \\
\text { diabetes }\end{array}$ & $95 \% \mathrm{CI}$ & $p$ value \\
\hline \multicolumn{7}{|l|}{ Adiponectin (per SD decrease) } \\
\hline Adjusted for age, sex & 2.27 & $1.66-3.11$ & $<0.0001$ & 1.87 & $1.32-2.67$ & 0.0005 \\
\hline Adjusted for age, sex, BMI & 1.87 & $1.36-2.57$ & 0.0001 & 1.76 & $1.23-2.52$ & 0.002 \\
\hline Adjusted for age, sex, BMI, and HOMA-IR & 1.44 & $1.04-1.97$ & 0.03 & 1.71 & $1.19-2.45$ & 0.004 \\
\hline \multicolumn{7}{|l|}{ Stratified analyses (adjusted for age, sex, BMI) } \\
\hline Insulin sensitive (HOMA-IR $<75$ th percentile) & 1.10 & $0.73-1.67$ & 0.64 & 1.34 & $0.88-2.03$ & 0.18 \\
\hline${ }^{\mathrm{a}}$ Insulin resistant (HOMA-IR $\geq 75$ th percentile) & 1.60 & $1.10-2.31$ & 0.01 & 2.34 & $1.16-4.73$ & 0.02 \\
\hline
\end{tabular}

${ }^{\mathrm{a}}$ Insulin resistance was defined as above study-specific 75 th percentile 


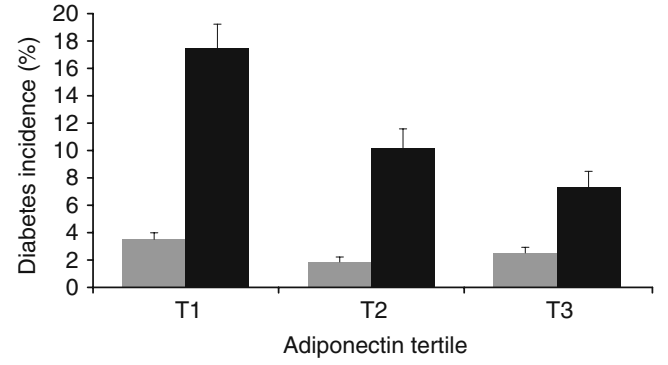

Fig. 1 Type 2 diabetes incidence over 6.5 year follow-up in each tertile of adiponectin, stratified by insulin resistance (HOMA-IR $<$ vs $\geq 75$ th percentile) in participants of the Framingham Offspring Study. Diabetes incidence in each tertile was adjusted for age and sex. Adiponectin level $(\mathrm{mg} / \mathrm{ml})$ ranges in each tertile: $\mathrm{T} 1=0.90-6.70$; $\mathrm{T} 2=6.80-11.50 ; \mathrm{T} 3=11.60-59.88$. Insulin-sensitive participants (HOMA-IR $<75$ th percentile), grey bars; insulin-resistant participants (HOMA-IR $\geq 75$ th percentile), black bars

type 2 diabetes (OR 1.49 [95\% CI 1.10-2.03]; $p=0.01$ ), while in the group without metabolic syndrome, the association was not significant (OR 1.22 [95\% CI 0.70 $2.13] ; p=0.47$ ), similar to analyses in stratified analyses of insulin-resistant vs insulin-sensitive individuals.

\section{Discussion}

We have demonstrated that lower adiponectin levels are associated with an increased risk of type 2 diabetes in participants of two population-based cohorts: the Framingham Offspring Study and the KORA study cohorts. This finding is in accordance with many previous reports in other prospective cohorts of various ethnic backgrounds as

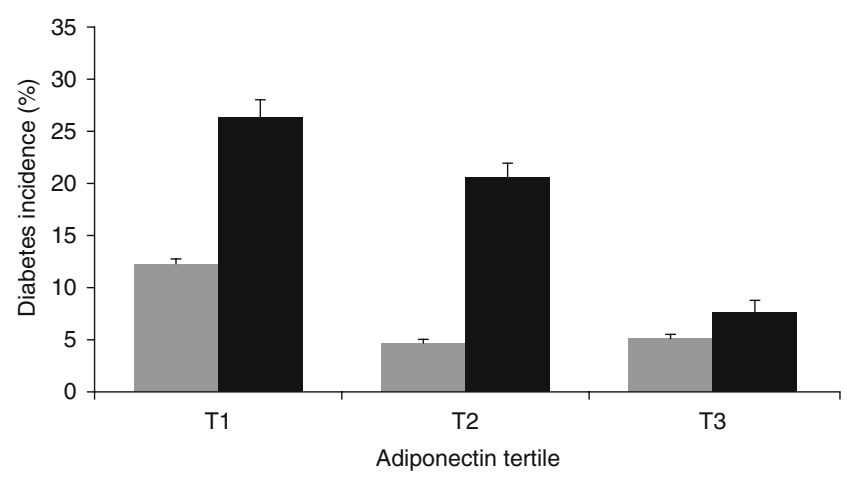

Fig. 2 Type 2 diabetes incidence over 8 year follow-up in each tertile of adiponectin, stratified by insulin resistance (HOMA-IR $<$ vs $\geq 75$ th percentile) in participants of the KORA S4/F4 study. Diabetes incidence in each tertile was adjusted for age and sex. Adiponectin level $(\mathrm{mg} / \mathrm{ml})$ ranges in each tertile: $\mathrm{T} 1=1.80-7.30, \mathrm{~T} 2=7.40-11.00$, $\mathrm{T} 3=11.10-89.90$. Insulin-sensitive participants (HOMA-IR $<75$ th percentile), grey bars; insulin-resistant participants (HOMA-IR $\geq 75$ th percentile), black bars reported in a recent meta-analysis [1]. A few studies have adjusted for insulin resistance measured by fasting insulin $[10,11]$ or HOMA-IR $[12,13]$; as in our analyses, the strength of associations was reduced by adjustment for insulin resistance but remained significant in most studies. We have further added to the understanding of the complex inter-relationships of insulin resistance and adiponectin and the risk of type 2 diabetes incidence by demonstrating that low adiponectin was associated with increased risk of developing type 2 diabetes only in insulin-resistant individuals and not in insulin-sensitive participants in two independent samples, even with a larger sample size in insulin-sensitive subgroups. This observation suggests that some degree of insulin resistance needs to be present to observe the association between low adiponectin and progression to diabetes. This is concordant with other reports where associations with adiponectin levels were stronger in individuals with higher metabolic risk profile. For example, Wannamethee et al. have reported that the association between adiponectin level and type 2 diabetes is stronger in individuals with higher BMI [13]. Moreover, we have previously shown that in Framingham Offspring Study participants, adiponectin levels are more strongly associated with insulin resistance in individuals with metabolic syndrome [9].

Low adiponectin levels have been suggested to be in the causal pathway leading to diabetes. In rhesus monkeys, adiponectin levels decrease in parallel with decreased insulin sensitivity, before the increase in glycaemic levels and diabetes incidence [14]. This does not preclude that some events early in the development of insulin resistance have to occur at the adipocyte level to decrease adiponectin secretion, which cannot then have its full beneficial action on peripheral tissues. In fact in our analysis of $A D I P O Q$ variants in the Framingham Offspring Study [2], we found that single nucleotide polymorphisms associated with low adiponectin levels were not associated with hyperglycaemia, once again suggesting that it is not necessarily a primary defect of adiponectin production or secretion that leads to type 2 diabetes.

Strengths and limitations Our study's strengths include the inclusion of two independent samples from well-established cohorts with large sample sizes, prospective designs and standardised measurements, including fasting blood samples for diagnosis of type 2 diabetes. We observed a significant interaction in KORA analyses, but not in Framingham; this may be because of a lower incidence of diabetes in Framingham. KORA participants were more at risk of diabetes at baseline (older, slightly heavier and more likely to have a positive family history) and were followed for a longer period, which results in a higher cumulative incidence than in Framingham. The lower incidence in Framingham 
might also be related to the fact that Framingham investigators did not perform OGTT at follow-up, leading to potential misclassification. HOMA-IR is a surrogate of insulin resistance, yet it was shown to be a fair way to classify participants in large cohorts such as here. However, HOMA-IR did not allow us to further investigate the contributions of hepatic and muscular insulin resistance on the interaction. Finally, both cohorts are composed of individuals who are mainly of white European descent and were at least of middle age when adiponectin levels were measured; our findings might not be generalisable to younger populations or those from different ancestral backgrounds.

\section{Conclusions}

We have demonstrated that low adiponectin levels are associated with an increased risk of developing diabetes in the Framingham Offspring Study and the KORA study, but only in individuals who are relatively insulin resistant. We believe that adiponectin has an important role in the pathophysiology of type 2 diabetes but our observation suggests that some event(s) prior to a decrease in adiponectin levels is necessary to induce the insulinresistant cascade leading to development of diabetes that is associated with low adiponectin. Longitudinal and repeated measures of both adiponectin levels and insulin resistance in humans will be helpful to clarify the sequence of events leading to type 2 diabetes.

Acknowledgements The study was supported by the National Heart, Lung and Blood Institute's Framingham Heart Study (Contract No. N01-HC-25195) the National Institutes of Health, National Center for Research Resources, NIH grants RO1 HL064753; RO1 HL076784; R01 AG028321; General Clinical Research Centers Program (Grant Number M01-RR-01066), and by a Career Development Award from the American Diabetes Association and NIDDK K24 DK080140 (J. B. Meigs). D. M. Nathan is supported in part by the Earle Charlton Fund for Innovative Diabetes Research. J. B. Meigs currently has research grants from GlaxoSmithKline, and has a consulting agreement with Interleukin Genetics. The KORA S4/F4 Diabetes Cohort Study was funded by a German Research Foundation project grant to W. Rathmann (DFG; RA 459/2-1). The German Diabetes Center is funded by the German Federal Ministry of Health, and the Ministry of School, Science and Research of the State of North-Rhine-Westfalia. The KORA research platform and the KORA Augsburg studies are financed by the Helmholtz Zentrum München, German Research Center for Environmental Health, which is funded by the German Federal Ministry of Education, Science, Research and Technology and by the State of Bavaria. We thank the field staff in Augsburg who were involved in the conduct of the studies.
Duality of interest The authors declare that there is no duality of interest associated with this manuscript.

\section{References}

1. Li S, Shin JJ, Ding EL, van Dam RM (2009) Adiponectin levels and risk of type 2 diabetes: a systematic review and meta-analysis. JAMA 302:179-188

2. Hivert MF, Manning AK, McAteer JB et al (2008) Common variants in the adiponectin gene (ADIPOQ) associated with plasma adiponectin levels, type 2 diabetes, and diabetes-related quantitative traits: the Framingham Offspring Study. Diabetes 57:3353-3359

3. Kannel WB, Gordon T, Castelli WP (1979) Obesity, lipids, and glucose intolerance. The Framingham Study. Am J Clin Nutr 32:1238-1245

4. Matthews DR, Hosker JP, Rudenski AS, Naylor BA, Treacher DF, Turner RC (1985) Homeostasis model assessment: insulin resistance and beta-cell function from fasting plasma glucose and insulin concentrations in man. Diabetologia 28:412-419

5. Rathmann W, Haastert B, Icks A et al (2003) High prevalence of undiagnosed diabetes mellitus in Southern Germany: target populations for efficient screening. The KORA survey 2000. Diabetologia 46:182-189

6. Rathmann W, Strassburger K, Heier M et al (2009) Incidence of Type 2 diabetes in the elderly German population and the effect of clinical and lifestyle risk factors: KORA S4/F4 cohort study. Diabet Med 26:1212-1219

7. Herder C, Hauner H, Haastert B et al (2006) Hypoadiponectinemia and proinflammatory state: two sides of the same coin?: results from the Cooperative Health Research in the Region of Augsburg Survey 4 (KORA S4). Diab Care 29:1626-1631

8. Expert Panel on Detection, Evaluation, and Treatment of High Blood Cholesterol in Adults (2001) Executive Summary of The Third Report of The National Cholesterol Education Program (NCEP) Expert Panel on Detection, Evaluation, And Treatment of High Blood Cholesterol In Adults (Adult Treatment Panel III). JAMA 285:2486-2497

9. Hivert MF, Sullivan LM, Fox CS et al (2008) Associations of adiponectin, resistin, and tumor necrosis factor-alpha with insulin resistance. J Clin Endocrinol Metab 93:3165-3172

10. Lindsay RS, Funahashi T, Hanson RL et al (2002) Adiponectin and development of type 2 diabetes in the Pima Indian population. Lancet 360:57-58

11. Duncan BB, Schmidt MI, Pankow JS et al (2004) Adiponectin and the development of type 2 diabetes: the atherosclerosis risk in communities study. Diabetes 53:2473-2478

12. Snehalatha C, Mukesh B, Simon M, ViswaNathan V, Haffner SM, Ramachandran A (2003) Plasma adiponectin is an independent predictor of type 2 diabetes in Asian Indians. Diab Care 26:3226-3229

13. Wannamethee SG, Lowe GD, Rumley A, Cherry L, Whincup PH, Sattar N (2007) Adipokines and risk of type 2 diabetes in older men. Diab Care 30:1200-1205

14. Hotta K, Funahashi T, Bodkin NL et al (2001) Circulating concentrations of the adipocyte protein adiponectin are decreased in parallel with reduced insulin sensitivity during the progression to type 2 diabetes in rhesus monkeys. Diabetes 50:1126-1133 